\title{
Hedgehog and Notch Signaling in Enteric Nervous System Development
}

\author{
Jessica Ai-jia Liu ${ }^{a} \quad$ Elly Sau-Wai Ngana, ${ }^{a}$ \\ ${ }^{a}$ Department of Surgery, and ${ }^{b}$ Centre for Reproduction, Development and Growth, Li Ka Shing \\ Faculty of Medicine, The University of Hong Kong, Pokfulam, Hong Kong, SAR, China
}

\author{
Key Words \\ Enteric nervous system $\cdot$ Neural crest cells $\cdot$ Hedgehog $\cdot$ Notch
}

\begin{abstract}
The enteric nervous system (ENS) in mammals is derived from a small pool of progenitor cells, namely enteric neural crest cells (NCCs). These precursor cells proliferate extensively to expand, migrate over a long distance to fully colonize the developing gut and differentiate into millions of neurons and glia to form a functional ENS for regulating the complex behaviors of the gut. This developmental process relies on a precise regulation of the neuronal and glial differentiation and requires an appropriate balance between the migration, proliferation and differentiation of enteric NCCs and their progeny. Hedgehog $(\mathrm{Hh})$ and Notch signalings are essential for almost every aspect of ENS development, and they confer both the long- and short-range signals to coordinate these seemingly diverse cellular processes. In this review, we summarize the roles of $\mathrm{Hh}$ and Notch signaling, particularly in the context of gut organogenesis and ENS development and emphasize how combinatory Hh and Notch signaling renders functional diversity as well as specificity.

(C) 2013 S. Karger AG, Basel
\end{abstract}

\section{Introduction}

The enteric nervous system (ENS) in mammals is derived from a small pool of enteric neural crest cells (NCCs). These precursor cells extensively proliferate to expand, migrate over a long distance to fully colonize the developing gut, and differentiate into millions of neurons and glia which are organized into a network to coordinate bowel movement. This developmental process relies on a precise regulation of the neuronal and glial differentiation, 
and requires an appropriate balance between the proliferation and differentiation of enteric NCCs and their progeny. Different combinations of long- and short-range instructive signals are implicated in various stages of NCC development that occur over a lengthy period of time. At early stages, these signals are involved in NCC specification and delamination and extend further into later stages for supporting gut colonization and formation of a functional neural network. As for ENS development, many of these signals originate from the gut mesenchyme, through the production of secretory molecules to activate their downstream molecular events in enteric NCCs that are distant from the origin of the signals. Alternatively, a short-range signaling is established where signaling molecules are secreted by enteric NCCs and target themselves or their neighboring cells. Hedgehog $(\mathrm{Hh})$ is one of the key morphogens expressed in gut mesenchyme, establishing various concentration gradients across the developing gut to offer the long-range regulation. Notch, on the other hand, is expressed by the enteric NCCs and acts on the adjacent cells functions through lateral inhibition or activation. Compelling evidence suggests that $\mathrm{Hh}$ and Notch signaling play equally important roles in ENS development. In this review, we focus on Hh and Notch signaling and emphasize how Hh and Notch work coordinately regulate ENS development.

\section{Enteric Nervous System}

ENS is mainly derived from vagal NCCs and partly from sacral NCCs. These progenitors move along the gut in a rostrocaudal manner and gradually colonize the developing gut. In addition to rostrocaudal migration, Nishiyama et al. [1] have recently shown that indeed a population of enteric NCCs migrates from the midgut to the hindgut via the mesentery (transmesenteric migration) and these transmesenteric NCCs contribute a large part of the hindgut ENS. Sacral NCCs, on the other hand, are only responsible for a small fraction of ENS in the distal midgut and hindgut, and their migration is apparently independent of that of the vagal NCCs [2]. While ENCCs are migrating, they also proliferate and progressively give rise to neurons and glia which are organized into ganglia and are localized in the two main plexuses. The outer myenteric plexuses are found between the longitudinal and circular muscle layers, and the inner submucosal plexuses reside within the submucosa of the intestine. These two plexuses are symmetrically interconnected to form a functional neuronal network to control motility, fluid exchange and gut hormone secretion $[3,4]$.

\section{Proliferation, Differentiation and Migration of Enteric NCCs}

Proper control of population size of pre-enteric NCCs is one of the key steps that determine their capacity to fully colonize the gut and to give rise to a full diversity of neurons and glia. When the total number of avian vagal NCCs was experimentally reduced by ablation of the dorsal neural tube in the vagal region, enteric NCCs failed to fully colonize the whole gut accompanied by a reduced number of neuronal subtypes [5]. Similarly, in mice, proliferation of enteric NCCs at the migratory wavefront was shown to be the main driving force for these cells to invade the intestine $[6,7]$. Therefore, correct population sizes of pre-enteric and enteric NCCs are critical for complete colonization of the gut.

Neurogenesis and gliogenesis are two highly coordinated developmental processes for generation of neurons and glia in ENS. Despite that there being a considerable overlap between the periods of enteric neurogenesis and gliogenesis, neurogenesis usually occurs first and overt gliogenesis is apparent slightly later. In mice, neuronal progenitors can be detected as early as E10-E10.5 just behind the migratory wavefront of the enteric NCCs [8]. 
These enteric progenitors generate new neurons throughout embryogenesis and even beyond P30. Nevertheless, only very limited neurogenesis occurs in the adult gut under steady-state conditions and adult ENS, unlike CNS, does not have constitutive neurogenesis by dividing progenitors $[9,10]$. The first glial precursors appear at around E11.5-E12 [11], and mature glial cells provide support and insulation for the enteric neurons, as well as being a scavenger to clear apoptotic cells [12]. More recently, it has been reported that neurogenesis occurs from a rare population of glial cells in the adult ENS upon injury induced by neurotoxic detergent (benzalkonium chloride) $[9,10]$. However, it is still not clear what kind of injuries might occur under physiological conditions that can induce neurogenesis of ENS. Overall, the adult ENS just has a low-level turnover of enteric glia under steady-state conditions as well as considerable regenerative capacity to form glia after injury, which would be crucial for the maintenance of ENS function.

\section{Hirschsprung's Disease}

Hirschsprung's (HSCR) disease is a congenital ENS defect and is attributed to a failure of enteric NCCs to colonize the hindgut, leading to lack of ganglion cells along varying segments of the intestine. HSCR patients usually exhibit abnormal bowel contraction or obstruction that leads to food bolus accumulation and severe constipation. HSCR exhibits multifactorial modes of inheritance and more than 14 genes have been identified associated with HSCR, but mutations in these genes are of incomplete penetrance and account for only $50 \%$ of the familial and up to $20 \%$ of the sporadic cases [13]. More recently, genome-wide association study (GWAS) conducted on HSCR patients has uncovered many more new disease loci and provided valuable insights into the contribution of structural variants to HSCR [14-17]. Intriguingly, many of these genetic variants/mutations are found in genes such as RET, NEUREGULIN 1 and 3, PATCHED1 and DELTA-LIKE3 that regulate the proliferation, migration or differentiation of enteric NCCs, highlighting the relevance of these genes in ENS development and HSCR pathogenesis. Till now, still little is known about the underlying mechanisms and how dysregulation of these genes may lead to HSCR disease. Therefore, the important next step is to convert novel genetic findings into the knowledge needed for a better understanding of disease mechanisms.

\section{Hh Signaling Pathway}

\section{Signal Transduction Machineries of Hh Pathway}

Hh is crucial for both embryogenesis and tumorigenesis in mammals and has been implicated in many human diseases. The Hh pathway can be activated by one of their three homologues of the Drosophila proteins, namely Sonic hedgehog (Shh), Desert hedgehog (Dhh) and Indian hedgehog (Ihh), of which Shh is the best characterized signaling protein involved in the early patterning and cell fate specification in various systems [18].

The fundamental machinery of $\mathrm{Hh}$ signal transduction involves three Gli proteins. For $\mathrm{Hh}$ signal activation, two transmembrane proteins including a receptor Patched (Ptch) and a signal activator Smoothened (Smo) are required. In the absence of Hh ligand, Ptch constitutively represses Smo activity. Upon binding of Hh, Ptch relieves its inhibition of Smo and activates downstream events. In mammals, membrane trafficking of Ptch and Smo is critical for Hh signaling. Receptor endocytosis of Ptch is implicated in shaping the signal gradient [19, 20], while Smo trafficking to the primary cilia is required for Hh signal transduction [21]. The downstream signal activation of Hh pathway is mainly mediated by a family of zinc-finger- 
containing transcriptional factors, Gli proteins (Gli1, Gli2 and Gli3) [22]. Gli1 and Gli2 function mainly as transcriptional activators, whereas Gli3 is a principle repressor. Both Gli2 and Gli3 are present in two forms: a full-length transcription activator $\left(\mathrm{Gli}^{\mathrm{A}}\right)$ and a truncated aminoterminal fragment with repressive activity $\left(\mathrm{Gli}^{\mathrm{R}}\right)$. In the absence of $\mathrm{Hh}, \mathrm{Gli} 2$ and Gli3 exist as a part of the protein complex in cytoplasm and are tethered to the microtubules. Proteolytic cleavage of the full-length Gli generates $\mathrm{Gli}^{\mathrm{R}}$, which enters the nucleus to suppress the $\mathrm{Hh}$ target genes. In the presence of $\mathrm{Hh}$, active Smo blocks the proteolysis of Gli and generate Gli activator $\left(\mathrm{Gli}^{\mathrm{A}}\right)$. Different $\mathrm{Hh}$ levels result in unique $\mathrm{Gil}^{\mathrm{A}} / \mathrm{Gli}^{\mathrm{R}}$ ratios, by which distinct sets of Hh target genes are switched on or off, conferring a gradient of Hh responses [23].

\section{Primary Cilium in Hh Signaling Transduction}

Primary cilium is a microtubule-based non-motile organelle that emanates from the cell surface of quiescent cells. It acts as a sensory organelle to coordinate various signaling pathways, which are critical in both embryonic development as well as tissue homeostasis in adulthood. Assembly of the cilia is mediated by intraflagellar transport (IFT), by which large complexes called IFT particles are moved from the basal body into and along the ciliary microtubules. Integrity of cilia is essential for mammalian $\mathrm{Hh}$ signal transduction [24]. As summarized in figure 1, in the absence of Hh ligand, the Ptch receptor resides in the ciliary membrane and prevents Smo from entering primary cilia. Upon stimulation by Hh ligand, Ptch exits the cilium and consequently triggers the ciliary accumulation of active Smo [25]. Translocation of Gli to the ciliary tip is also induced by Hh signal, so Gli proteins accumulate at ciliary tips after pathway activation. Furthermore, active Smo progressively activates the full-length Gli proteins to $\mathrm{Gli}^{\mathrm{A}}$ and induces the microtubule-dependent translocation of Gli to the nucleus to activate transcription of Hh target genes [26, 27]. Defective ciliary assembly or function results in an attenuated Shh pathway, leading to an array of congenital diseases in humans.

\section{Hh Signaling Components in the Developing Gut}

In all mammals examined, $S h$, Ihh and Dhh are expressed in the developing gut tube. $S h h$ and $I h h$ are expressed in the epithelium, while $D h h$ is specifically expressed in Schwann cells, peripheral nerves and endothelial cells. In mouse, during gut organogenesis, $S h h$ and Ihh are co-expressed in gut primitive endoderm epithelium at early somite stage $(8.5 \mathrm{dpc})$ before the enteric NCCs reach the stomach that usually occurs at E10.5. At the later stages, from the early stomach development (E10) to the initiation of epithelial cytodifferentiation at E15.5, Shh and Ihh are persistently, but differentially expressed in the developing guts. Shh remains its high level expression in the forestomach and lower level in the hindgut, whereas Ihh expression is more restricted to the posterior stomach. By E14.5, Shh expression in the small intestine is downregulated while Ihh expression remains. In E18.5 gut, Shh expression appears to be re-upregulated and highly expressed in the glandular stomach, small intestine, and colon, but its expression is mostly restricted to colonic crypts [28]. Expression of Ptch1 is localized in the mesoderm and mirrors that of $S h h$, but not for $I h h$. There is only very low expression of Ptch1 in the distal stomach, it suggests that Ihh may not be translated or remains inactive at early stage. More recently, the temporal and spatial expression of $\mathrm{Hh}$ components including Ptch1, Gli1, Gli2, and Gli3 are better defined with various Gli reporter mouse lines. Mo et al. [29] have nicely demonstrated the dynamic alteration of Hh activity in the stomach and intestine, further supporting the presence of high Hh activity domain in the stomach versus low $\mathrm{Hh}$ activity in the intestine. Importantly, the expressions of the other key Hh signal transduction machineries such as Ptch1, Smo, Gli1, Gli2, and Gli3 have demonstrated in enteric NCCs, highlighting the relevance of Hh signal in the ENS development [16, 30]. 


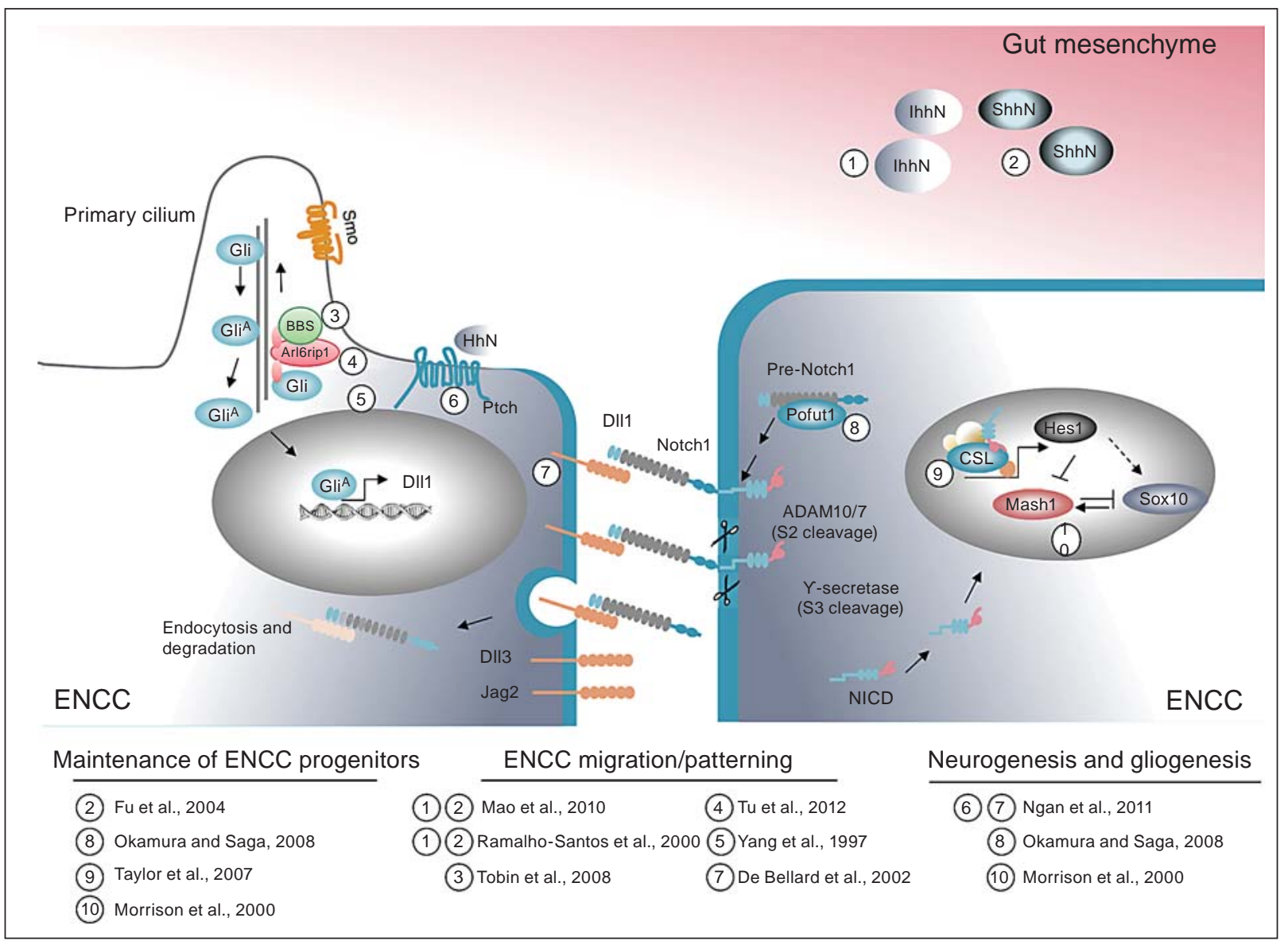

Fig. 1. Hh and Notch signal transduction in enteric NCC (ENCC). Upon binding of Hh to Ptch, Smo is unleashed from Ptch. Ptch is subjected to internalization and active Smo accumulates in the primary cilia, which induces the translocation of Gli proteins to the tip of the primary cilium by intraflagellar transport (IFT). BBS and Arl6rip may regulate the ciliary trafficking of Gli. Active Smo also activates the full-length Gli proteins to form $\mathrm{Gli}^{\mathrm{A}}$. Gli ${ }^{\mathrm{A}}$ then moves to the nucleus by the microtubule-dependent transport system and triggers gene transcription. Delta ligands (Dll1 and Dll3) are expressed in the surface of signaling sending NCCs, and subsequently bind to the Notch receptor which is expressed in the neighboring cells. Upon binding of ligand to the receptor, the receptor is subjected to two proteolytic cleavages (S2 and S3 cleavages) mediated by ADAM10/7 and $\gamma$-secretase, respectively, and lead to generation of free Notch intracellular domain (NICD). NICD enters the nucleus and interacts with CSL (e.g. RBP-J) to activate the transcription of Notch target genes, such as Hes family genes. GDP-fucose protein O-fucosyltransferase 1 (Pofut1) is essential for the cell surface expression of Notch receptor. Hh and Notch pathway genes implicated in various aspects of ENS development are summarized in the lower panel.

\section{Hh Signaling in Enteric NCC Development}

Implications of Hh signaling in ENS development have been directly demonstrated in various model systems. In the zebrafish, Shh expression is first detected at the anterior end of the intestine, while Ptch1 is widely expressed throughout the mesenchyme adjacent to the endoderm and is expressed by enteric NCCs. Shh is essential for the migration of enteric NCCs from the vagal region to the anterior end of the intestine in both the pre-enteric phase and an enteric phase. No migrating enteric NCCs were observed when Hh signaling was attenuated either by cyclopamine or in the hedgehog pathway homozygous mutants: sonic you (syu), that has a deletion in the Shh gene, and smooth muscle-omitted (smu), that has a mutation in 
zebrafish Smo. In the pre-enteric phase, Shh likely acts either directly or indirectly, via regulating the production of glial cell line-derived neurotrophic factor (Gdnf), to direct the vagal neural crest-derived enteric NCC to the anterior end of the intestine. However, during the enteric phase of ENS development, the key activity of Shh on ENS precursors is as a mitogen rather than as an indirect regulator of ENS precursors' migratory ability [31].

Similarly, in the avian system, Shh secreted from the endodermal epithelium is also essential for the gut development. Inhibition of the Shh signaling by cyclopamine results in an ectopic formation of enteric neurons accompanied by an increased differentiation of smooth muscle cells in chick gut explants. Particularly, the severity of the patterning defect and aganglionosis is positively correlated to the concentration of cyclopamine being used, suggesting the relevance of the graded activity of $\mathrm{Hh}$ in enteric neuron formation $[32,33]$.

Hh signaling in mouse ENS development is also indispensable and mouse mutants carrying mutations in various Hh components consistently exhibit ENS defects as summarized in figure 1. Both $S h h^{-/-}$and $I h h^{-/-}$mouse mutants exhibit severe ENS defects despite with distinct phenotypes. In Shh null, enteric NCCs are still able to migrate along and fully colonize the developing gut. However, ectopic formation of ganglions is observed in the stomach and small intestine, where a substantial number of neurons are found differentiating abnormally in the epithelium of villi. $I \mathrm{hh}^{-/-}$mutants, on the other hand, exhibit totally different phenotypes, in which $50 \%$ of the mutants display HSCR-like phenotypes with absence of ganglions in the distal regions of colon [28]. The incomplete penetrance of the phenotypes suggests that other factors or modifier genes are likely involved in determining the severity of the phenotypes. Given the overlapping distribution and functional redundancy between Shh and Ihh, removal of both Shh and Ihh is required in order to precisely define the function of Hh proteins in gut organogenesis and ENS development. $\mathrm{Sh}^{-/-} ; \mathrm{Ih}^{-/-}$double mutant mouse displays more severe phenotypes which dies at E8.5, precluding an analysis of ENS development. More recently, the $\mathrm{Cre} / \mathrm{lox} P$ system has been applied to delete $\mathrm{Sh} h$ and $\mathrm{Ih} h$ specifically in the early gut endoderm. Mutants with conditional ablation of Shh and $I h h$ consistently exhibit severe ENS phenotypes. Despite the fact that some enteric neurons still exist in the stomach at E12.5 and E16.5, the number of neurons is noticeably diminished [34]. The analyses of single and double mutants reflect that Shh and Ihh are critical for ENS development, but probably in different aspects. It is also important to note that the N-terminal signaling peptides of Shh and Ihh exhibit more than $90 \%$ amino acid sequence homology, and both bind to Ptch 1 and activate the Hh signaling cascades of the responsive cells with a similar mechanism. However, how inactivation of these two similar genes leads to such different phenotypes and what are the underlying mechanisms for Shh and Ihh to differentially regulate ENS development remain to be fully elucidated.

Cell autonomous action of $\mathrm{Hh}$ in enteric NCCs has also been demonstrated using the enteric NCC culture, where Shh negatively regulates the migration and neuronal differentiation of enteric NCCs. Pretreatment of enteric NCCs with Shh abolishes Gdnf-induced neuronal differentiation and migration [30]. A subsequent study demonstrates that Shh indeed only transiently promotes the proliferation of enteric NCCs, while constitutive activation of the $\mathrm{Hh}$ pathway by deletion of Ptch1 or long-term treatment with Shh or its agonist (purmorphamine) favors the differentiation of enteric NCCs towards the glial lineage. Conditional ablation of Ptch1 in NCCs results in premature gliogenesis and a reduced number of ENS progenitors, through activation of the Notch signaling pathway. Intriguingly, epistasis analysis of genetic data generated from a GWAS on HSCR patients further reveals that PTCH1 represents a susceptibility gene for HSCR disease and cumulative effects of variations in PTCH1 and DLL3 confer a higher risk to HSCR. This study has defined a potential disease mechanism that glial mediators may contribute to the pathogenesis of HSCR [16].

Gli1, Gli2 and Gli3 are the transcription factors mediating Hh signaling output. Multiplicity of Gli proteins allows a two-tiered control of Hh signal transduction, where Gli2 and 
Gli3 are the primary transducers, and Gli1 acts as the secondary transducer refining $\mathrm{Hh}$ signaling. Like in other systems, Gli1 is a direct Hh target gene in enteric NCCs and activation of Hh signaling by deletion of Ptch1 or addition of Shh or its agonist robustly upregulates Gli1 expression [16]. Although $\mathrm{Gli1}^{-/-}$mice do not show any severe developmental defect [35, 36], the implication of Gli1 in ENS development has been demonstrated in an early study with ectopic overexpression of human GLI1 gene in transgenic mice. Transgenic mice of ectopic expression of GLI1 display a HSCR-like phenotype and severity of the phenotype is directly associated with the expression level of GLI1 transgene [38]. In addition, Gli1 expression is strictly regulated by Gli2 and Gli3 in early mouse embryo, so Gli2 and/or Gli3 are likely the key transcription factor(s) for Hh activity. The expression pattern of Gli2 is similar to that of Gli1, but the expression level of Gli2 transcripts in enteric NCCs is low and no obvious upregulation of Gli2 has been detected upon Hh stimulation in vitro [unpubl. data]. In addition, with the mouse reporter line the Gli2 signal is barely detectable in enteric neurons at E14.5 in mice [37]. Thus, the role of Gli2 in ENS development is still elusive. Gli3, on the other hand, is highly expressed in the enteric NCCs including both activator $\left(\mathrm{Gli}^{\mathrm{A}}\right)$ and repressor $\left(\mathrm{Gli}^{\mathrm{R}}{ }^{\mathrm{C}}\right.$ ) forms. As deletion of Ptch1 in enteric NCCs results in a reduced expression of Gli3 ${ }^{\mathrm{R}}$ [16], the primary role for Hh signaling activity is likely to inhibit the formation of $\mathrm{Gli}^{\mathrm{R}}{ }^{\mathrm{i}}$ in enteric NCCs.

Recent advances in gene targeting have deepened our knowledge about Hh signaling and the presence has been suggested of distinct Hh activity domains in the developing gut of a high Hh level in the stomach versus a low level in the intestine. The differential Hh activity domains may result in the formation of a distinct Gli activator to repressor ratio $\left(\mathrm{Gli}^{\mathrm{A}} / \mathrm{Gli}^{\mathrm{R}}\right)$ in the stomach versus intestine, such that Hh would further confer the context-dependent gene regulation and generate the graded $\mathrm{Hh}$ responses along the developing gut. Different experimental approaches that specifically remove or overexpress the $\mathrm{Gli}^{\mathrm{A}}$ or $\mathrm{Gli}^{\mathrm{R}}$ will be necessary to unambiguously reconcile whether graded Hh signaling plays any patterning role during colonization of stomach and intestine.

The second line of evidence supporting the involvement of Hh signaling in ENS development relies on the close association between ENS defects and various Shh-dependent craniofacial defects. Bardet-Biedl syndrome (BBS) is a primary ciliopathy with IFT dysfunction and characterized by facial dysmorphology. Twelve BBS genes (BBS1-BBS12) have been identified so far and mutations in these genes cause primary cilia dysfunction [39]. HSCR disease represents a component feature associated with BBS, so it has been suggested that these two diseases share a common genetic basis. Studies in zebrafish indicated that Bbs 8 morphant embryos are reminiscent of Shh pathway mutants or cyclopamine-treated fish embryos and exhibit a blunted response to Hh stimuli of a significant reduction of Ptch1 and Gli1 expression accompanied by an accumulation of $\mathrm{Gli}^{\mathrm{R}}$. These Hh-deficient fishes recapitulate human disease phenotypes and display both craniofacial and ENS defects [40]. Concordant data has been obtained recently by Tu et al. [41]. They have knocked down a major intracellular trafficking regulator called ADP ribosylation factor-like 6 interacting protein 1 (Arl6rip1) in zebrafish and the Arl6rip1 morphant fishes display HSCR-associated BBS-like phenotypes. Loss of Arl6rip1 dampens Shh signaling and leads to migration defects of vagal NCCs, resulting in incomplete colonization of the gut.

\section{Notch Signaling}

Signal Transduction Machineries of the Notch Pathway

Notch signaling is highly conserved among different species with a well-defined framework. Like Hh, Notch is one of the most pivotal developmental signals in mammals and aberrant Notch signaling has been implicated in varying human diseases. Four Notch receptors 
(Notch1-4) and their ligands Delta (Delta1-4) and Serrate/Jagged (Jagged1-2) are transmembrane domain proteins. Upon ligand stimulation, Notch receptor will undergo proteolytic cleavage by two steps - S2 and S3 cleavages. S2 cleavage is mediated by the ADAM family, followed by a second cleavage through the $\gamma$-secretase complex (S3 cleavage). These cleavages lead to the generation of a free intracellular domain (NICD), which subsequently translocates into the nucleus and binds to the DNA-binding proteins CSL (e.g. Su(H), CBF and RBP-Jk) to initiate the transcription of various target genes including Hes1. Hes1 is a transcriptional repressor and it negatively regulates its downstream targets, such as Mash1, neurogenin (Ngn) or MyoD to maintain progenitor cells from differentiation [42] (fig. 1).

\section{Notch Signaling Components in the Developing and Adult Guts}

The expressions of Notch signaling components in mouse developing gut have been well described. At E11.5, Notch1, Dll1 and Dll3 are expressed in the majority of enteric NCCs, but only few cells express Notch4, Dll4 and Jag [43]. Notch1-3 is highly expressed in the endoderm and mesenchyme at E12.5, but Notch4 is absent at these stages. Higher Hes1 expression is detected in endoderm when compared to its expression in mesenchyme, indicating a more active Notch signaling is present in the endoderm. Notch ligands Jag1 and Dll3 are also expressed in the mesenchymal cells [44]. In the rat adult ENS, Notch1 and Jag2 are specifically expressed in non-proliferating ganglion cells located in the myenteric and submucosal plexuses. Notch1 expression is detected only in one half of all myenteric neurons. It is preferentially expressed in the cholinergic neurons, but is absent in the nitric oxide synthase and calretinin-immunoreactive neurons. Jag2 expression, on the other hand, is detected in most of the neurons [45]. This observation raises a possibility that Notch1 has a role in specifying and maintaining cholinergic neurons, while Jag2 functions in the maintenance of all types of neuronal cells in the adult ENS. It is also consistent with an observation that reduced expression of NOTCH1 and JAG2 are detected in the aganglionic segments of HSCR bowel [46].

\section{Notch Signaling in Enteric NCC Development}

Deletion of Notch1, Notch2, Jag1, or Dll1 in mouse are embryonic lethal and mutants die at the early stage before ENS development starts [47-50], while Notch3, Notch4, or Dll3 null show mild phenotypes and no obvious NC-associated defect is observed [51-53]. Subsequent studies using conditional genetic ablation of different Notch signaling components have clearly dissected the peculiar roles of Notch signaling in various aspects of ENS development (table 1). For instance, genetic ablation of $R b p j$ in mouse NCCs causes defects in glial specification or differentiation leading to a significant reduction in number of enteric glia and neurons. Interestingly, at E10.5, comparable numbers of enteric progenitors were found in the mutant and control guts and the mutants do not show any depletion of pre-migratory NCC progenitors, suggesting that loss of Rbpj does not affect the capability of enteric NCCs to colonize the gut. However, a significant reduction in the number of neurons and glia is observed at E14.5 and this reduction persists through to E18.5. In addition, Taylor et al. [54] show that Rbpj-deficient enteric NCCs exhibit a similar proliferation rate in vivo, but the frequency of mutant cells to form multiple lineage colonies in culture is threefold lower than the control. Therefore, it is possible that Rbpj may not only be required for the glial lineage differentiation but also for the maintenance of undifferentiated ENS progenitors. Notch signaling is also implicated in NCC migration, where deletion of Dll1 leads to defects in NCC migration. Dll1-deficient NCCs lose their rostral-caudal polarity and the mutant cells are randomly distributed rather than following the normal migratory path [55]. A more recent study with conditional gain of Notch signaling (Wnt1Cre;Rosa ${ }^{\text {Notch }}$ ) in NCCs has revealed that the aberrant Notch signaling leads to ENS defects through interrupting the migratory pathway of NCCs. Ectopic activation of Notch signaling in the pre-migratory NCCs disrupts the normal 
Table 1. ENS phenotypes in Hedgehog and Notch mouse mutants

\begin{tabular}{|c|c|c|}
\hline Mutants & Phenotypes of ENS & Reference \\
\hline $\mathrm{Shh}^{-/-}$ & $\begin{array}{l}\text { Reduction of smooth muscle } \\
\text { Ectopic formation of enteric neurons } \\
\text { Disruption of ENS plexus }\end{array}$ & {$[28]$} \\
\hline$I h h^{-/-}$ & $\begin{array}{l}\text { Reduction of smooth muscle } \\
\text { Lack of neurons } \\
\text { Aganglionosis of distal colon }\end{array}$ & {$[28]$} \\
\hline$S h h^{C r e / f} ; I h h^{-/ f}$ & Significantly reduction of neurons & {$[34]$} \\
\hline Wnt1-Cre; Ptch-1/f & $\begin{array}{l}\text { Reduction of enteric NCC progenitors } \\
\text { Premature glial differentiation }\end{array}$ & {$[16]$} \\
\hline GLI1 transgenic mice & $\begin{array}{l}\text { Malformation of smooth muscle layer of the gastrointestinal wall } \\
\text { Significant reduction of myenteric plexuses }\end{array}$ & {$[38]$} \\
\hline Wnt1-Cre; RBP-j $\mathrm{J}^{\mathrm{f} / \mathrm{f}}$ & $\begin{array}{l}\text { Defective migration of enteric NCC } \\
\text { Decreased proliferation } \\
\text { Reduction of enteric NCCs in the midgut } \\
\text { No glia formation }\end{array}$ & {$[54,56]$} \\
\hline Wnt1-Cre; Rosa ${ }^{\text {Notch }}$ & $\begin{array}{l}\text { Increased proliferation } \\
\text { Disruption of NCC migratory route } \\
\text { Absence of enteric NCC in midgut }\end{array}$ & {$[56]$} \\
\hline Wnt1-Cre; Pofut1 ${ }^{\mathrm{f} / \mathrm{f}}$ & $\begin{array}{l}\text { Premature neurogenesis } \\
\text { Reduction of glial progenitors (loss of Sox10) }\end{array}$ & {$[43]$} \\
\hline Delta-1-/- & $\begin{array}{l}\text { Depletion of the enteric NCCs precursor pool } \\
\text { Abnormal migratory route }\end{array}$ & {$[55]$} \\
\hline
\end{tabular}

migratory route of NCCs, in which migrating NCCs are organized into two distinct streams but instead migrate in a bulk population. Although the enteric NCCs of Wnt1Cre;Rosa ${ }^{\text {Notch }}$ mutants still retain their capacity to colonize the gut, the number of enteric NCCs is markedly reduced and there is almost complete absence of NCCs in the midguts of these mutants [56]. In the context of NCC differentiation, Notch signaling is also indispensable and implicated in both inhibiting neurogenesis and promoting the gliogenesis. Treatment of enteric NCCs with Dll1 robustly induces Notch receptor cleavage accompanied by accumulation of NICD and Hes1, leading to the elevated expression of various glial markers, such as S100 $\beta$ and GFAP and loss of neurogenic plasticity. Furthermore, Notch signaling likely subserves a similar role in mouse and human. NCCs isolated from human skin, also called skin-derived progenitors (SKPs), show similar propensity to give rise to cells expressing glial markers when they are exposed to DLL1 [16]. Similarly, when the Notch signal is inhibited, enteric NCCs undergo premature neurogenesis. Protein 0-fucosyltransferase 1 (Pofut1) functions upstream of NICD and is responsible for the Notch-Delta interactions and trafficking of the Notch receptor [57, 58]. Mouse mutants with NCC-specific ablation of Pofut1 show a premature neurogenesis of enteric NCCs and reduced glial progenitors. Moreover, Sox10 expression is profoundly downregulated in the Pofut1 mutant embryos at E11.5 and is even completely absent at E12.5, implying that Notch signaling promotes gliogenesis through mediating Sox10 expression [43]. Intriguingly, Notch and Sox10 contribute to both the maintenance of ENS progenitors and promoting gliogenesis, an additional regulatory mechanism is likely involved in different 
developmental stages to confer the dual functions of Notch and Sox10 in these two processes. In addition to positive regulation of Sox10 expression to promote gliogenesis, Notch also simultaneously upregulates Hes1 which subsequently downregulates Mash1 expressions to inhibit neurogenesis. As a negative feedback, the regulatory mechanism between Sox10 and Mash1 has been reported where Sox10 induces Mash1 expression and Mash1 re-suppresses the Sox10 expression [60]. Notch signaling is therefore likely involved in balancing the Sox10 and Mash 1 activities to enable a continuous derivation of mature neurons and glia while maintaining a consistent progenitor population [56, 61].

\section{Combinatorial Action of Hh and Notch Signaling in the Embryonic Gut}

The temporal and spatial expression regulation of Hh and Notch ligands and their receptors, graded Hh activity or lateral inhibition/induction of Notch signaling, multiplicity of transcription factors or proteolytic signaling transduction cascades, all confer multiple layers of signal confinement for $\mathrm{Hh}$ and Notch signalings. Indeed, combinatorial action of these two signals may further refine the cellular response, where combinations of exposure to these signals would be contemporaneous or occur sequentially.

Interaction between $\mathrm{Hh}$ and Notch has been evidenced in various stages of gut development. Firstly, Notch signaling components and their receptors are expressed in close proximity to or co-expressed in the Hh-responsive cells of the gut mesenchyme as well as in the enteric NCCs. The second evidence showing the potential interactions between these two prominent signals is based on the fact that Hh and Notch share the common downstream targets such as Hes1 and Hes5 [44]. More recently, Kim et al. [44] have shown that mouse mutants with excessive Notch or lack of Hh signaling develop similar defects in the stomach mesenchyme, where a significant reduction of enteric neurons and loss of nearly all lamina propria fibroblasts are found. Concordant with these observations, Hh-deficient embryos also show Notch overactivity in their defective gut mesenchyme and exposure to recombinant Shh override Notch-induced death of cultured fetal gut mesenchymal cells [34, 44]. All these data support the notion that endodermal $\mathrm{Hh}$ signaling regulates the development of gut mesenchyme at least in part through restraining the level of Notch activity. Regarding the ENS development, Notch and Hh signaling are found interacted at both genetic and functional levels and contribute to ENS development and HSCR pathogenesis. Ngan et al. [16] have performed a canonical correlation analysis (CCA) on the data obtained from GWAS with HSCR patients. The data suggests that a combination of certain PTCH1 and DLL3 alleles confers a higher risk to HSCR. Subsequent functional study using mouse mutants with NCC-specific ablation of Ptch1 further indicates that constitutive activation of Hh leads to a robust activation of Notch signaling through inducing Dll1 expression. As a consequence of excessive $\mathrm{Hh}$ and Notch signal, the number of enteric progenitors is markedly reduced and associated with premature gliogenesis. This study has highlighted how multiple signals may integrate and lead to diverse cellular responses. In general, Hh signaling acts as a mitogen to promote the cell proliferation, but reduced proliferation of enteric progenitors is observed in the Ptch1-/mutants. It would probably be the secondary effect of premature gliogenesis where the Notch signal is likely the key mediator for this process. Therefore, Hh may work cooperatively with Notch signaling to confine the temporal regulation of enteric NCC development including proliferation, the onset of neuronal differentiation and the switch from neurogenesis to gliogenesis [16]. 


\section{Conclusion}

ENS development relies on the coordination between the enteric NCCs and gut mesenchyme. Mesenchymal signals confer a long-range regulation, acting either on the enteric NCCs or muscle to directly or indirectly control the formation of the neuronal network in the bowel. The short-range regulation primarily refers to the local signals expressed in the enteric NCCs that act laterally to regulate the neighboring cells. Intriguingly, many of these signals have reiterated functions. For instance, while Shh and Notch are essential for maintaining the progenitor pool at the early stages, Shh activates the Notch signal to initiate glial differentiation at the later phases of ENS development. Combination of the long- and short-range signals provides an additional layer of signal refinement. To date, understanding how these signalings are differentially regulated and utilized to program the development of enteric NCC still remains a major unresolved question. Establishment of the causal relationship between the external stimuli and the transcription factor hierarchies for NCC development may help dissect the complexity of these signaling networks.

\section{Acknowledgement}

This work was supported by a research grant HKU775710 from the Hong Kong Research Grants Council to E.S.-W.N.

\section{References}

1 Nishiyama C, Uesaka T, Manabe T, et al: Trans-mesenteric neural crest cells are the principal source of the colonic enteric nervous system. Nat Neurosci 2012;15:1211-1218.

2 Wang X, Chan AK, Sham MH, et al: Analysis of the sacral neural crest cell contribution to the hindgut enteric nervous system in the mouse embryo. Gastroenterology 2011;141:992-1002.e1-6.

- 3 Obermayr F, Hotta R, Enomoto H, et al: Development and developmental disorders of the enteric nervous system. Nat Rev Gastroenterol Hepatol 2013;10:43-57.

- 4 Heanue TA, Pachnis V: Enteric nervous system development and Hirschsprung's disease: advances in genetic and stem cell studies. Nat Rev Neurosci 2007;8:466-479.

- 5 Peters-van der Sanden MJ, Kirby ML, Gittenberger-de Groot A, et al: Ablation of various regions within the avian vagal neural crest has differential effects on ganglion formation in the fore-, mid- and hindgut. Dev Dyn 1993;196:183-194.

6 Landman KA, Simpson MJ, Newgreen DF: Mathematical and experimental insights into the development of the enteric nervous system and Hirschsprung's disease. Dev Growth Differ 2007;49:277-286.

7 Simpson MJ, Zhang DC, Mariani M, et al: Cell proliferation drives neural crest cell invasion of the intestine. Dev Biol 2007;302:553-568.

- 8 Young HM, Ciampoli D, Hsuan J, et al: Expression of Ret-, p75(NTR)-, Phox2a-, Phox2b-, and tyrosine hydroxylase-immunoreactivity by undifferentiated neural crest-derived cells and different classes of enteric neurons in the embryonic mouse gut. Dev Dyn 1999;216:137-152.

9 Laranjeira C, Sandgren K, Kessaris N, et al: Glial cells in the mouse enteric nervous system can undergo neurogenesis in response to injury. J Clin Invest 2011;121:3412-3424.

10 Joseph NM, He S, Quintana E, et al: Enteric glia are multipotent in culture but primarily form glia in the adult rodent gut. J Clin Invest 2011;121:3398-3411.

11 Young HM, Bergner AJ, Muller T: Acquisition of neuronal and glial markers by neural crest-derived cells in the mouse intestine. J Comp Neurol 2003;456:1-11.

12 Gershon MD: Behind an enteric neuron there may lie a glial cell. J Clin Invest 2011;121:3386-3389.

13 Emison ES, Garcia-Barcelo M, Grice EA, et al: Differential contributions of rare and common, coding and noncoding Ret mutations to multifactorial Hirschsprung disease liability. Am J Hum Genet 2010;87:60-74.

14 Tang CS, Ngan ES, Tang WK, et al: Mutations in the NRG1 gene are associated with Hirschsprung disease. Hum Genet 2012;131:67-76.

15 Tang CS, Cheng G, So MT, et al: Genome-wide copy number analysis uncovers a new HSCR gene: NRG3. PLoS Genet 2012;8:e1002687.

-16 Ngan ES, Garcia-Barcelo MM, Yip BH, et al: Hedgehog/Notch-induced premature gliogenesis represents a new disease mechanism for Hirschsprung disease in mice and humans. J Clin Invest 2011;121:3467-3478. 
17 Garcia-Barcelo MM, Tang CS, Ngan ES, et al: Genome-wide association study identifies NRG1 as a susceptibility locus for Hirschsprung's disease. Proc Natl Acad Sci USA 2009;106:2694-2699.

18 Chiang C, Litingtung Y, Lee E, et al: Cyclopia and defective axial patterning in mice lacking Sonic hedgehog gene function. Nature 1996;383:407-413.

19 Incardona JP, Lee JH, Robertson CP, et al: Receptor-mediated endocytosis of soluble and membrane-tethered Sonic hedgehog by Patched-1. Proc Natl Acad Sci USA 2000;97:12044-12049.

20 Torroja C, Gorfinkiel N, Guerrero I: Patched controls the Hedgehog gradient by endocytosis in a dynamindependent manner, but this internalization does not play a major role in signal transduction. Development 2004;131:2395-2408.

21 Goetz SC, Anderson KV: The primary cilium: a signalling centre during vertebrate development. Nat Rev Genet 2010;11:331-344.

22 Hui CC, Slusarski D, Platt KA, et al: Expression of three mouse homologs of the Drosophila segment polarity gene cubitus interruptus, Gli, Gli-2, and Gli-3, in ectoderm- and mesoderm-derived tissues suggests multiple roles during postimplantation development. Dev Biol 1994;162:402-413.

23 Hui CC, Angers S: Gli proteins in development and disease. Annu Rev Cell Dev Biol 2011;27:513-537.

-24 Huangfu D, Liu A, Rakeman AS, et al: Hedgehog signalling in the mouse requires intraflagellar transport proteins. Nature 2003;426:83-87.

25 Rohatgi R, Milenkovic L, Scott MP: Patched1 regulates hedgehog signaling at the primary cilium. Science 2007; 317:372-376.

26 Humke EW, Dorn KV, Milenkovic L, et al: The output of Hedgehog signaling is controlled by the dynamic association between Suppressor of Fused and the Gli proteins. Genes Dev 2010;24:670-682.

27 Tukachinsky H, Lopez LV, Salic A: A mechanism for vertebrate Hedgehog signaling: recruitment to cilia and dissociation of SuFu-Gli protein complexes. J Cell Biol 2010;191:415-428.

-28 Ramalho-Santos M, Melton DA, McMahon AP: Hedgehog signals regulate multiple aspects of gastrointestinal development. Development 2000;127:2763-2772.

29 Mo R, Kim JH, Zhang J, et al: Anorectal malformations caused by defects in sonic hedgehog signaling. Am J Pathol 2001;159:765-774.

30 Fu M, Lui VC, Sham MH, et al: Sonic hedgehog regulates the proliferation, differentiation, and migration of enteric neural crest cells in gut. J Cell Biol 2004;166:673-684.

-31 Reichenbach B, Delalande JM, Kolmogorova E, et al: Endoderm-derived Sonic hedgehog and mesoderm Hand2 expression are required for enteric nervous system development in zebrafish. Dev Biol 2008;318:52-64.

32 Sukegawa A, Narita T, Kameda T, et al: The concentric structure of the developing gut is regulated by Sonic hedgehog derived from endodermal epithelium. Development 2000;127:1971-1980.

-33 Fukuda K, Yasugi S: Versatile roles for sonic hedgehog in gut development. J Gastroenterol 2002;37:239-246.

-34 Mao J, Kim BM, Rajurkar M, et al: Hedgehog signaling controls mesenchymal growth in the developing mammalian digestive tract. Development 2010;137:1721-1729.

-35 Bai CB, Stephen D, Joyner AL: All mouse ventral spinal cord patterning by hedgehog is Gli dependent and involves an activator function of Gli3. Dev Cell 2004;6:103-115.

-36 Park HL, Bai C, Platt KA, et al: Mouse Gli1 mutants are viable but have defects in SHH signaling in combination with a Gli2 mutation. Development 2000;127:1593-1605.

37 Kolterud A, Grosse AS, Zacharias WJ, et al: Paracrine Hedgehog signaling in stomach and intestine: new roles for hedgehog in gastrointestinal patterning. Gastroenterology 2009;137:618-628.

38 Yang JT, Liu CZ, Villavicencio EH, et al: Expression of human GLI in mice results in failure to thrive, early death, and patchy Hirschsprung-like gastrointestinal dilatation. Mol Med 1997;3:826-835.

-39 Karaman A: Bardet-Biedl syndrome: a case report. Dermatol Online J 2008;14:9.

-40 Tobin JL, Di Franco M, Eichers E, et al: Inhibition of neural crest migration underlies craniofacial dysmorphology and Hirschsprung's disease in Bardet-Biedl syndrome. Proc Natl Acad Sci USA 2008;105:6714-6719.

41 Tu CT, Yang TC, Huang HY, et al: Zebrafish arl6ip1 is required for neural crest development during embryogenesis. PLoS One 2012; 7:e32899.

42 Bray SJ: Notch signalling: a simple pathway becomes complex. Nat Rev Mol Cell Biol 2006; 7:678-689.

43 Okamura Y, Saga Y: Notch signaling is required for the maintenance of enteric neural crest progenitors. Development 2008;135:3555-3565.

44 Kim TH, Kim BM, Mao J, et al: Endodermal Hedgehog signals modulate Notch pathway activity in the developing digestive tract mesenchyme. Development 2011;138:3225-3233.

-45 Sander GR, Brookes SJ, Powell BC: Expression of Notch1 and Jagged2 in the enteric nervous system. J Histochem Cytochem 2003;51:969-972.

46 Jia H, Zhang K, Chen Q, et al: Downregulation of Notch-1/Jagged-2 in human colon tissues from Hirschsprung disease patients. Int J Colorectal Dis 2012;27:37-41.

47 Swiatek PJ, Lindsell CE, del Amo FF, et al: Notch1 is essential for postimplantation development in mice. Genes Dev 1994;8:707-719.

48 Hamada Y, Kadokawa Y, Okabe M, et al: Mutation in ankyrin repeats of the mouse Notch2 gene induces early embryonic lethality. Development 1999;126:3415-3424.

49 Xue Y, Gao X, Lindsell CE, et al: Embryonic lethality and vascular defects in mice lacking the Notch ligand Jagged1. Hum Mol Genet 1999;8:723-730. 
50 Hrabe de Angelis M, McIntyre J 2nd, Gossler A: Maintenance of somite borders in mice requires the Delta homologue DII1. Nature 1997;386:717-721.

51 Krebs LT, Xue Y, Norton CR, et al: Characterization of Notch3-deficient mice: normal embryonic development and absence of genetic interactions with a Notch1 mutation. Genesis 2003;37:139-143.

52 Krebs LT, Xue Y, Norton CR, et al: Notch signaling is essential for vascular morphogenesis in mice. Genes Dev 2000;14:1343-1352.

53 Dunwoodie SL, Clements M, Sparrow DB, et al: Axial skeletal defects caused by mutation in the spondylocostal dysplasia/pudgy gene Dll3 are associated with disruption of the segmentation clock within the presomitic mesoderm. Development 2002;129:1795-1806.

54 Taylor MK, Yeager K, Morrison SJ: Physiological Notch signaling promotes gliogenesis in the developing peripheral and central nervous systems. Development 2007;134:2435-2447.

55 De Bellard ME, Ching W, Gossler A, et al: Disruption of segmental neural crest migration and ephrin expression in delta-1 null mice. Dev Biol 2002;249:121-130.

-56 Mead TJ, Yutzey KE: Notch pathway regulation of neural crest cell development in vivo. Dev Dyn 2012;241: 376-389.

57 Okajima T, Irvine KD: Regulation of notch signaling by O-linked fucose. Cell 2002;111:893-904.

$\$ 58$ Okajima T, Xu A, Irvine KD: Modulation of notch-ligand binding by protein O-fucosyltransferase 1 and fringe. J Biol Chem 2003;278:42340-42345.

59 Oka C, Nakano T, Wakeham A, et al: Disruption of the mouse RBP-J kappa gene results in early embryonic death. Development 1995;121:3291-3301.

60 Kim J, Lo L, Dormand E, et al: SOX10 maintains multipotency and inhibits neuronal differentiation of neural crest stem cells. Neuron 2003;38:17-31.

61 Morrison SJ, Perez SE, Qiao Z, et al: Transient Notch activation initiates an irreversible switch from neurogenesis to gliogenesis by neural crest stem cells. Cell 2000;101:499-510. 